\title{
LINKAGE AND THE EVOLUTION OF DOMINANCE
}

\author{
W. J. EWENS \\ Department of Statistics, Australian National University, Canberra
}

Received 5.ix.65

\section{INTRODUCTION}

THE present author has recently re-examined mathematically the theory of Fisher (1928a, $b, \mathrm{I} 929, \mathrm{I} 958)$ that the phenomenon of dominance is a result of natural selection of modifying genes. The general conclusion of this examination is that the theory requires some reconsideration, since several formulae used by Fisher, and hence possibly the conclusions based on them, are false. For details the reader is referred to Ewens $(1965 a, b)$.

A discussion of the relative importance of the various factors governing the evolution of dominance process was given in the second of these papers. It will be noted that one factor was not considered, namely that of linkage between the primary and the modifying locus. While it was not doubted that linkage would have some effect on the process, it was not felt that it would be an important factor. On the other hand, Bodmer and Parsons (1962), in a discussion of the effect of linkage in evolutionary processes, conclude that "modifiers of the fitness of a heterozygote at a locus $A-a$ would be selected far more rapidly if they were linked to this locus", and that " a modifier $B$ whose only effect is to enhance the heterozygote advantage of $A a$ will only be selected if it is sufficiently closely linked to the locus $A-a$ ". This conclusion was predicted on general arguments in an earlier paper (Parsons and Bodmer, I96I) and supported by a mathematical argument in the latter paper. On this view linkage is clearly regarded as a most important factor, and the purpose of the present paper is to investigate the difference of opinion between this view and that implied by (Ewens, $1965 a, b)$.

\section{NUMERICAL RESULTS}

The theory of the evolution of dominance concerns two loci, namely the locus " $A$ " of primary interest, at which occur alleles $A$ and $a$, and a modifying locus " $M$ ", at which occur alleles $M$ and $m$, whose effect is to alter the expression of the heterozygote $A a$ if the modifying allele $M$ is present at this locus. A mathematical analysis of such a process cannot treat the primary and modifying loci separarely, but must treat both loci jointly and simultaneously. It is by now well known (Moran (1964), Bodmer and Parsons (Ig62), Lewontin and Kojima (196o)) that for such a joint process it is necessary to consider the frequencies $c_{1}, c_{2}, c_{3}$ and $c_{4}$ of the gametes $A M, a M, A m$ and $a m$ respectively, rather 
than the frequencies $q_{A}$ and $q_{M}$ of the genes $A$ and $M$, since the latter are not sufficient to give a complete description of the process. The behaviour of the process in terms of gamete frequencies is as follows. Using the notation of Moran (1964) we shall suppose that the fitnesses of the nine possible genotypes are given by the following array:

$\begin{array}{lccc} & A A & A a & a a \\ M M & w_{11} & w_{12} & w_{13} \\ M m & w_{21} & w_{22} & w_{23} \\ m m & w_{31} & w_{32} & w_{33}\end{array}$

Note that we assume that the coupling and repulsion double heterozygotes have the same fitness. The present analysis can be extended immediately to the case where this is not so, but such extension is not the main concern of this discussion. The frequencies $c_{1}^{\prime}, c_{2}^{\prime}, c_{3}^{\prime}$ and $c_{4}^{\prime}$ of the gametes $A M, a M, A m$ and $a m$ in the next generation are given in terms of those at the present generation by the equations

$W c_{1}^{\prime}=w_{11} c_{1}^{2}+w_{12} c_{1} c_{2}+w_{21} c_{1} c_{3}+w_{22} c_{1} c_{4}+R w_{22}\left(c_{2} c_{3}-c_{1} c_{4}\right)$, $W c_{2}^{\prime}=w_{12} c_{1} c_{2}+w_{13} c_{2}^{2}+w_{8_{2}} c_{2} c_{3}+w_{23} c_{2} c_{4}-R w_{22}\left(c_{2} c_{3}-c_{1} c_{4}\right)$, $W c_{3}^{\prime}=w_{21} c_{1} c_{3}+w_{22} c_{2} c_{3}+w_{31} c_{3}^{2}+w_{32} c_{3} c_{4}-R w_{22}\left(c_{2} c_{3}-c_{1} c_{4}\right)$, $W c_{4}^{\prime}=w_{22} c_{1} c_{4}+w_{23} c_{2} c_{4}+w_{32} c_{3} c_{4}+w_{33} c_{4}^{2}+R w_{22}\left(c_{2} c_{3}-c_{1} c_{4}\right)$.

Here $R$ is the coefficient of recombination between the $A$ and $M$ loci and $\mathbb{W}$, the mean fitness of the population, is given by the expression

$$
\begin{array}{r}
\bar{W}=w_{11} c_{1}^{2}+2 w_{12} c_{1} c_{2}+w_{13} c_{2}^{2}+2 w_{21} c_{1} c_{3}+2 w_{22}\left(c_{1} c_{4}+c_{2} c_{3}\right) \\
+2 w_{23} c_{2} c_{4}+w_{31} c_{3}^{2}+2 w_{32} c_{3} c_{4}+w_{33} c_{4}^{2} .
\end{array}
$$

The rough reasoning which led the present author to regard linkage as being relatively unimportant in an evolution of dominance process can be sketched as follows. Whereas the equations

$$
c_{1}=q_{A} q_{M}, \ldots, c_{4}=\left(\mathrm{I}-q_{A}\right)\left(\mathrm{I}-q_{M}\right),
$$

are not generally satisfied, in a wide variety of cases they are approximately true, at least within an order of magnitude, (see, for instance, the numerical example in Moran (1964)). If equations (2) and (3) are added, and if equations (2) and (4) are added, we find that

and

$$
\Delta q_{M}=c_{1}^{\prime}+c_{2}^{\prime}-c_{1}-c_{2}
$$

$$
\Delta q_{A}=c_{1}^{\prime}+c_{3}^{\prime}-c_{1}-c_{3}
$$

do not depend explicitly on the recombination fraction $R$. This does not mean that these quantities are completely independent of $R$, since $c_{1}, c_{2}, c_{3}$ and $c_{4}$ will themselves depend on $R$. If, however, the equations (7) hold, joint consideration of (7) and (8) shows that $\Delta q_{M}$ and $\Delta q_{A}$ are quite independent of $R$. Thus if the equations (7) are approximately true, then $\Delta q_{M}$ and $\Delta q_{A}$ will be approximately independent of $R$. But the arguments which have been considered for the evolution of dominance process concern the relative rates of change of $q_{M}$ and $q_{A}$, 
so that the rough conclusion was drawn that in such a process linkage would not be an important factor.

Such reasoning is, of course, rather rough and we now discuss the question in more detail. It is supposed that at the primary locus $A$, the allele $A$ has a selective advantage over $a$, so that in the absence of any modifying genes the fitnesses of $A A, A a$, and $a a$ are I, $1-s h, \mathrm{I}^{-s}$ respectively, where $s>0,0<h \leqq \mathrm{I}$. The effect of the modifying gene $M$ is to increase the fitness of the heterozygote $A a$ towards that of the homozygote $A A$. We may suppose that such modification depends on whether $M$ occurs in a homozygote $M M$ or a heterozygote $M m$. Specifically, we shall assume that the fitnesses of the various genotypes are given by the following array:

$\begin{array}{lcll} & A A & A a & a a \\ M M & \text { I } & \text { I } & \text { I }-s \\ M m & \text { I } & \text { I }-s k & \text { I }-s \\ m m & \text { I } & \text { I }-s h & \text { I }-s\end{array}$

where o $\leqq k \leqq h \leqq \mathrm{I}$.

We now consider two problems associated with the process. The first problem concerns the situation where the fitnesses given in (9) have been in force for some time. In this case the allele $a$ will occur only because of recurrent mutation from $A$ to $a$, and the problem is to find a formula for the increase $\Delta q_{M}$ of the frequency of the modifier $M$ under these conditions. This has been discussed at some length in Ewens (1965a) and here we only consider this problem so far as it concerns linkage. Using an incorrect method, Fisher (1929b) found, for the case $k=0$, the formula

$$
\Delta q_{M}=2 u q_{M}
$$

for the rate of increase in the frequency of $M$, where $u$ is the mutation rate $A \rightarrow a$. This formula was regarded as being important since it indicated that $\Delta q_{M}$ increases monotonically as $q_{M}$ increases to unity. This contrasts with the behaviour of the expression

$$
\Delta q_{M}=K q_{M}\left(\mathrm{I}-q_{M}\right)
$$

which holds for " normal " selection pressures, so that if $q_{M}$ is sufficiently close to unity the dominance modification selection pressure would exceed that due to other factors. The present author showed that in fact $\Delta q_{M}$ behaved like $\left(\mathrm{I}-q_{M}\right)^{2}$ near $q_{M}=\mathrm{I}$, so that selection pressure due to dominance modification is in fact very minor and is less than that due to other selection pressures near $q_{M}=\mathrm{I}$. For our present purposes we merely note that it was also shown that this result holds irrespective of the degree of linkage between the $A$ and $M$ loci. To this extent, linkage is of secondary importance in the evolution of dominance.

A second problem, which is our main concern, is to consider what happens if initially the $A$ allele is rare. This would happen if initially the allele $a$ had a selective advantage over $A$, and then due perhaps to a 
changing environment the allele $A$ develops a selective advantage over $a$ and the fitnesses become as shown in (9). This problem is relevant because, in the first problem considered above, the increase $\Delta q_{M}$ of the modifier is of the order of magnitude of the mutation rate $u$ from $A$ to $a$ (it may sometimes be of the order of magnitude of $\sqrt{u}$ ), and is therefore small (quite apart from the factor $\left(1-q_{M}\right)^{2}$ ). This occurs because under the conditions of the first problem the frequency of the heterozygote $A a$ is of the order of magnitude of the mutation rate $u$, and because the selective advantage of the modifier $M$, depending as it does on modification of this rate heterozygote, is correspondingly small. If, on the other hand, $q_{A}$ changed from near zero to near unity there will be a large number of heterozygotes $A a$ upon which the dominance modification process can act. This means that $\Delta q_{M}$ will generally be much larger than in the first problem. The real question is to find how much $q_{M}$ changes as $q_{A}$ goes from zero to unity and to consider the effect of linkage on this change.

We shall do this by considering numerical cases, since an exact analysis using equations (2)-(5) is very difficult.

The initial values of $q_{M}$ and $q_{A}$ are rather arbitrary. Since the allele $A$ has a selective disadvantage up to the time the process starts, the modifier $M$ will also be at an (induced) selective disadvantage. On Fisher's theory we should then take the initial frequency of $M$ to be small. The following initial values for the gamete frequencies were therefore chosen as being reasonable:

$$
c_{1}=0.000 \mathrm{I}, \quad c_{2}=0.0099, \quad c_{3}=0.0099, \quad c_{4}=0.980 \mathrm{I} .
$$

The effect of linkage between primary and modifying locus will depend to some extent on the values assumed by the constants $h, k$ and $s$. If $s$ is small, all the fitnesses $w_{11} \ldots w_{33}$ are close to unity, in which case (cf. Moran (1964)) the equations (7) will be fairly good as approximations. In this case equations (2)-(5) show that the effect of linkage is not very great. If $s$ large we expect linkage to be more important.

In order to obtain more detailed information, a computer programme was run which gave values of $c_{1}, c_{2}, c_{3}$ and $c_{4}$ in successive generations, using the recursion formulae (2)-(5). The programme was designed to run until the frequency $c_{1}+c_{3}$ exceeded 0.95 , printing out meanwhile values of $c_{1}, c_{2}, c_{3}$ and $c_{4}$ every five generations. For our purposes we shall consider only the value of $q_{M}=c_{1}+c_{2}$ (found by interpolation) at the time when $q_{A}=c_{1}+c_{3}$ reached 0.95 . These values are given in table I below for various values of $R$ and $s$, the values of $h$ and $k$ being fixed at $\mathrm{I}, 0.5$ respectively.

Inspection of table I verifies the general argument outlined above. For $s=0.5$ the effect of linkage is much more marked than for $s=0 \cdot \mathrm{I}$, and for any value of $s$, increasing the degree of linkage increases the change in frequency of the modifier $M$.

It may, however, be questioned whether the increase in the modifier demonstrated by table $\mathrm{I}$ is affected by linkage to a significant degree. 
Once the frequency $q_{A}$ of the primary allele $A$ is close to unity, and is kept less than unity only by recurrent mutation from $A$ to $a$, the discussion of the first problem above becomes relevant. It will be useful for us to refer to the period when $q_{A}$ increases from near zero to near unity as Phase $I$, and to the period after this as Phase 2. Consider, for example, the cases $s=0 . \mathrm{I}, R=0.5$ and $s=0 . \mathrm{I}, R=0.0 \mathrm{I}$. In the former the frequency $q_{M}$ will only increase to about 0.3 at the end of Phase I, while in the latter $q_{M}$ increases to about 0.9 . During stage 2 further change in the frequency of $M$ will be determined by three factors, namely the selective advantage of $M$ due to dominance modification, "other" selective advantages of $M$, and random sampling.

TABLE I

Values of $\mathrm{q}_{\mathrm{M}}\left(=\mathrm{c}_{1}+\mathrm{c}_{2}\right)$ when the value of $\mathrm{q}_{\mathrm{A}}\left(=\mathrm{c}_{1}+\mathrm{c}_{3}\right)$ reached 0.95 , found from the relations (2)-(5), for indicated values of $\mathrm{R}$ and $\mathrm{s}$. The number of generations required is given in brackets below the value of $\mathrm{q}_{\mathrm{M}}$ for each case

\begin{tabular}{|c|c|c|}
\hline$R$ & $s=0.1$ & $s=0.5$ \\
\hline & & \\
\cline { 2 - 3 } 0.01 & 0.861 & 0.942 \\
& $(276)$ & $(35)$ \\
0.03 & 0.668 & 0.913 \\
0.05 & $(314)$ & $(39)$ \\
& 0.505 & $0.88 \mathrm{I}$ \\
0.10 & $(393)$ & $(41)$ \\
& 0.347 & 0.801 \\
0.20 & $(531)$ & $(42)$ \\
& 0.294 & 0.648 \\
0.30 & $(581)$ & $(44)$ \\
0.40 & 0.282 & 0.515 \\
& $(595)$ & $(50)$ \\
0.50 & 0.276 & 0.427 \\
& $(601)$ & $(57)$ \\
& 0.273 & 0.378 \\
& $(604)$ & $(62)$ \\
\hline
\end{tabular}

If the population is large we can ignore the last factor. Now the rate of change of $q_{M}$ due to "other" selective advantages of $M$ is of the form

$$
\Delta q_{M}=K q_{M}\left(\mathrm{I}-q_{M}\right)
$$

where $K$ is a constant which we may take to be equally likely to be positive or negative, and of absolute value perhaps of the order $0 \cdot 00 \mathrm{I}$ to $O \cdot I$. The rate of change of $q_{M}$ due to dominance modification is rather difficult to obtain but is approximately given by

$$
\Delta q_{M}=u q_{M}\left(\mathrm{I}-q_{M}\right)
$$

where $u$ is the mutation rate from $A$ to $a$. Under an argument analogous to that used in deriving (Io) we would derive the incorrect expression

$$
\Delta q_{M}=u q_{M}
$$

for this change. Now using (14) it might be arguable that if $q_{M}$ is sufficiently large, selection pressure due to dominance modification 
would exceed that due to "other" causes (since the latter approaches zero as $q_{M}$ approaches unity) and the modifier would become fixed. In this way linkage would be a relevant factor since, for example, at the end of Phase I (14) might exceed (12) for $q_{M}=0.9$ but not for $q_{M}=0.3$. Under the correct formula (13), however, such an argument would not hold and the selection pressure (12) would probably always majorise the selection pressure (13) due to dominance modification. Thus if $K$ were negative the frequency of $M$ would steadily decrease during Phase 2, while if $K$ were positive the frequency of $M$ would steadily increase and dominance of $A$ over $a$ would result. However, this could hardly be ascribed to the dominance modification process itself but rather to the fact that the modifier $M$ happened to be advantageous of its own accord, and would occur equally irrespective of whether after Phase I, $q_{M}$ took the values 0.9 or 0.3 .

In small populations, on the other hand, the effect of random sampling should be considered and an allele having frequency 0.9 after Phase I would have a greater chance of fixation than an allele having frequency 0.3 . In this case, then, it might be argued that linkage during Phase I may be an important factor. On the other hand, if the population size is small, random sampling during Phase I will have considerable effect and may well have an effect as large as that of linkage (for an example of the effect of random sampling in small populations during Phase I, see Crosby (1963)).

To sum up, the effect of linkage, treated deterministically, is to increase the frequency of the modifier after Phase I by a factor of 2 or 3 compared with the unlinked case. This will probably not be important in large populations, since for large populations what is important during Phase 2 is not the frequency of the modifier at the beginning of this phase, but the selective advantage of the modifier. This is equally likely to be positive or negative. For small populations, the frequency of the modifier at the beginning of Phase 2 is important, but it does not follow that linkage during Phase I will necessarily give large frequencies of the modifier at this stage since random sampling effects are likely to be of the same order of magnitude as linkage effects.

We note that this increase is rather less than the "far more rapid" increase predicted by Bodmer and Parsons ( 1962 ), and also that contrary to their assertion the modifier always increases in frequency irrespective of the degree of linkage (this is revealed by more detailed results not presented here). Linkage does not have a qualitative effect but only a moderate quantitative effect, and there is no value $R_{0}$ of the recombination fraction such that the modifier increases in frequency only if $R<R_{0}$. In order to discuss their argument at greater length we turn now to their mathematical analysis.

\section{MATHEMATICAL ANALYSIS}

We firstly summarise the argument which led Bodmer and Parsons to their conclusion that linkage is an important factor in an evolution of 
dominance process. It is assumed in their argument that $c_{1}, c_{2}, c_{3}$, and $I-c_{4}$ are initially all small, which is the case considered for the numerical discussion in the previous section. If, then, we ignore "second order" terms like $c_{1}^{2}, c_{1} c_{2}, c_{1}\left(\mathrm{I}-c_{4}\right)$, etc., equations (2) and (4) assume the "linearised" forms

$$
c_{1}^{\prime}=w_{22} c_{1}(\mathrm{I}-R) / w_{33}
$$

and

$$
c_{3}^{\prime}=\left\{w_{32} c_{3}+R w_{22} c_{1}\right\} / w_{33} .
$$

From these linearised equations it is possible to write down explicitly the values $c_{1}^{(n)}$ and $c_{3}^{(n)}$ of $c_{1}$ and $c_{3}$ after $n$ generations in terms of the initial values $c_{1}^{(o)}$ and $c_{3}^{(o)}$. Doing this we get

$$
\begin{aligned}
& c_{1}^{(n)}=\left\{w_{22}(\mathrm{I}-R) / w_{33}\right\}^{n} c_{1}^{(o)} \\
& c_{3}^{(n)}=\left\{\frac{w_{22}(\mathrm{I}-R)}{w_{33}}\right\}^{n}\left\{\frac{R w_{22} c_{1}^{(o)}}{w_{22}(\mathrm{I}-R)-w_{32}}\right\} \\
& +\left\{\frac{w_{32}}{w_{33}}\right\}^{n}\left\{\frac{R w_{22} c_{1}^{(o)}}{w_{32}-w_{22}(\mathrm{I}-R)}+c_{\mathrm{s}}^{(o)}\right\}
\end{aligned}
$$

These equations are precisely the same as equation 5 on page 74 of Bodmer and Parsons ( 1962 ) if allowance is made for notational differences. From equations ( $\mathrm{I} 7$ ) and (I8) the conclusion is drawn that if $w_{22}(I-R)>w_{33}$, then $c_{1}$ will increase geometrically in frequency, and if $R<\left(w_{22}-w_{32}\right) / w_{22}$ the increase in $c_{1}$ will be geometrically faster than that in $c_{3}$ (since $c_{3}^{(o)}$ is generally much larger than $c_{1}^{(o)}$ ). It is quite clear, on this argument, that the behaviour of $c_{1}$ and $c_{3}$, both absolutely and relatively, depends crucially on the value of $R$, which is why so much importance is placed on linkage by Bodmer and Parsons.

This argument is, I believe, rather misleading, the reason being that it is not valid to form the linearised equations ( 15 ) and (16) from (2) and (4). This is so because although $c_{1}, c_{2}, c_{3}$ and $\mathrm{I}-c_{4}$ are certainly all small, they are not of the same order of smallness. In fact the product $c_{2} c_{3}$, involving two small terms, will generally be of the same order of magnitude as $c_{1} c_{4}$, involving only one small term. It is therefore not allowable to ignore $c_{2} c_{3}$ in comparison with $c_{1} c_{4}$, which is essentially what is done in forming the linearised equations (I5) and (I6).

To illustrate this we consider the case $w_{22}=0.95, w_{33}=0.9$, $R=0.5$. Then equation ( 17 ) gives

$$
c_{1}^{(n)}=(0.5278)^{n} c_{1}^{(o)}
$$

suggesting that $c_{1}$ decreases very rapidly with $n$. This is not in fact what happens; the numerical results indicate that $c_{1}$ increases monotonically. The same argument holds for $c_{3}$ and shows that the criteria $w_{22}(\mathrm{I}-R)>w_{33}$ and $R<\left(w_{22}-w_{32}\right) / w_{22}$ are meaningless. 


\section{SUMMARY}

The problem of linkage between the primary and modifying loci in an evolution of dominance process is considered. The conclusion is drawn that linkage may be of moderate importance, and that it is rather more important than was implied in Ewens $(1965 a, b)$ but rather less important than stated by Bodmer and Parsons (1962). The general view of the author expressed in the two papers referred to above is that dominance is less likely to develop from an evolutionary process than was thought by Fisher, and this view is not seriously changed by the results of this paper. Ewens.

Acknowledgment.-The computer programmes were written and run by Mr. P. M.

\section{REFERENCES}

BODMER, W. F., AND PARSONS, P. A. (1962). "Linkage and recombination in evolution." Advanc. Genet., II, I-гоo.

GrosBy, J. L. ( $\left(g_{63}\right)$. " The evolution and nature of dominance." $\mathcal{F}$. Theoret. Biol., $5,35-5 \mathrm{I}$.

EWENS, W. J. ( $1965 a)$. "A note on Fisher's theory of the evolution of dominance." To appear in Ann. Hum. Genet.

EwENs, w. J. ( $\left(\mathrm{g}^{6} 5_{5} b\right)$. "Further notes on the evolution of dominance." Heredity, $20,443-450$.

FISHER, R. A. (1928a). "The possible modification of the response of the wild type to recurrent mutations." Amer. Nat., 62, I I 5-I 26.

FISHER, R. A. (1928b). " Two further notes on the origin of dominance." Amer. Nat., $62,57 \mathrm{I}-574$.

FISHER, R. A. (1929). "The evolution of dominance; reply to Professor Sewell Wright." Amer. Nat., 63, 553-556.

FISHER, R. A. (1958). "The Genetical Theory of Natural Selection." (Second edition). Dover, New York.

LEWONTIN, R. C., AND KOJIMA, K. (I96o). "The evolutionary dynamics of complex polymorphisms." Evolution, I4, 458-472.

MORAN, P. A. P. (1964). "On the nonexistence of adaptive topographies." Ann. Hum. Genet., 27, 383-393.

PARSONS, P. A., AND BODMER, W. F. (Ig6I). "The evolution of overdominance: natural selection and heterozygote advantage." Nature, Igo, 7-12.

wright, s. (I929a). "Fisher's theory of dominance." Amer. Nat., 63, 274-279.

WRIGHT, s. (I929b). "The evolution of dominance. Comment on Dr. Fisher's reply." Amer. Nat., 63, 556-56r.

WRIGHT, s. (I934). "Physiological and evolutionary theories of dominance." Amer. Nat., 68, 24-53. 